\title{
Sense in Order: Channel Selection for Sensing in Cognitive Radio Networks
}

\author{
Ying Dai and Jie Wu \\ Department of Computer and Information Sciences \\ Temple University, Philadelphia, PA 19122 \\ Email: \{ying.dai, jiewu\}@temple.edu
}

\begin{abstract}
This paper considers a pre-phase of spectrum sensing in cognitive radio networks (CRNs), which is about how to choose a channel for spectrum sensing. We take the time dimension, spectrum dimension, and spacial dimension into account and propose a sense-in-order model. In this model, each node maintains four states regarding each channel, based on the neighbors' shared information. We construct a state transition diagram for the four states and design an algorithm for every node to calculate the probability of choosing each channel. Moreover, we extend our model by introducing the angle dimension into the sense-in-order (SIO) model. In the extended model, each node is equipped with a four-directional antenna, which creates more channel opportunities. We describe the problem that is brought about by directional antennas, and propose a weight-based solution for each node to determine the channel state in each direction. Extensive simulation results testify for the performance of our model.
\end{abstract}

Index Terms-Cognitive radio networks, directional antenna, sense-in-order, spectrum sensing.

\section{INTRODUCTION}

Cognitive radio networks (CRNs) [1] are a promising solution to the channel (spectrum) congestion problem. Primary users in CRNs are privileged users, for whom transmission is free from interference. Each node (secondary user) in a CRN is capable of sensing the available channels and can make opportunistic use of them without causing interference to primary users. During the process, one of the most important phases is the spectrum sensing.

A lot of work has been done on how to achieve an accurate result through spectrum sensing [2]-[4]. Each time a secondary user needs to find one channel for transmission, it will pick one channel for sensing. If the channel is unavailable, it needs to adjust its parameters and switch to sense another channel. For example, in Fig. 1, there is a pair of primary users, $T X$ and $R X$. The secondary user $u$ is in the interference range (the amoeba shape) of $T X$, who is using the channel $m_{1}$ to send data to $R X$. There is a total of three channels $\left(m_{1}, m_{2}, m_{3}\right)$ in the network. If $u$ needs to use one channel, it will pick one channel from the three channels. Because there are no differences among the three channels from $u$ 's point of view, it is possible that $u$ will pick $m_{1}$ for sensing. Then, after $u$ finds out that $m_{1}$ is unavailable based on the sensing results, it must switch to another channel to sense again. However, if $u$ can have some information about the three channels before sensing, it may avoid $m_{1}$ and select other channels for sensing.

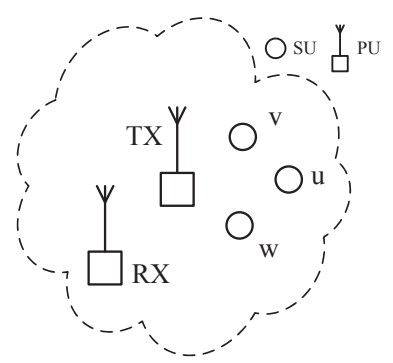

Fig. 1. Sense-in-order can reduce delay and save energy.

To some extent, both the delay and the energy consumption are able to be reduced.

Our focus here is not the spectrum sensing technology itself. Rather, we consider how to choose a channel for sensing for each node at the beginning, so that the probability of switching to sense another channel is reduced. This is also called the prephase of spectrum sensing. We propose a sense-in-order (SIO) model, which provides each node with an order for spectrum sensing. The order is determined before the spectrum sensing, and is maintained as a list by each node. When a node needs to find a channel for data transmission, it can look up the list and select a channel that has a higher probability of being available for sensing. In this way, each node knows the order to sense, which results in a reduction of switches among channels during spectrum sensing.

To determine the order of channels for sensing, both the space and time dimensions are considered. Firstly, the space factor influence lies in that nodes in a similar geographical area usually share similar channel information. One node can broadcast its sensing results to other nodes. Secondly, the time dimension is also very important, due to the dynamics of primary users. For each node, the channel information received more recently is more likely to be accurate. Based on the information from both dimensions, we identify four different states and their transitions for every channel, which is maintained on each node. Based on the states of different channels, each node is able to divide the whole channel into several different subsets. Each subset is assigned with a probability of being chosen. We also take conflict avoidance into account, since nodes in close locations are very likely to choose the same channel for sensing.

Most previous models in CRNs assume that the primary and secondary users transmit in all directions. In fact, with the 


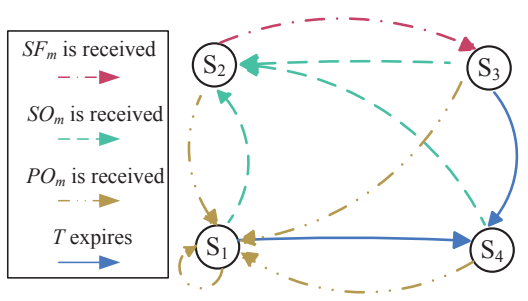

Fig. 2. State transition diagram.

developments regarding the directional antenna, more channel opportunities can be created if the angle dimension can be taken into account for spectrum sensing. This is because the primary and secondary users are able to share the same channel, even in the close geographical area. Therefore, based on the above SIO model, we propose a more complex model, in which each node is equipped with a four-directional antenna.

The main contributions of our paper are:

- We propose an SIO model for the pre-phase of spectrum sensing, which determines the order of selecting channels to sense for each node, which can reduce the delay and energy cost during the spectrum sensing phase.

- We identify the possible states for every channel on each node and define the state transitions, based on the signal that the node receives and the valid time period.

- We improve our model by considering the angle dimension. We make use of four-directional antennas and show how to determine the order for sensing on each direction.

\section{RELATED WORKS}

Our related works section is made up of two parts. The first part is spectrum sensing in CRNs. The second part is about the applications that use directional antennas.

Cooperative sensing is studied in [2], [5]-[7]. They focus on the spectrum sensing phase, which is based on the collaboration among different secondary users. However, our model is about how to select channels for sensing before the spectrum sensing phase, in order to reduce the costs of energy and delay. A recommendation-based model is proposed in [8], [9]. The authors are inspired by the recommendation system for e-commerce, and they have each node recommend channels to other nodes for spectrum sensing. However, their model only considers the appearance of primary users in the recommended channels. In fact, channel availabilities depend not only on primary users, but also on secondary users. Our model considers four different situations in each channel, which reduces the amount of conflicts among secondary users.

Different antenna models have been proposed in wireless networks [10], [11]. Primarily, they are about the neighbor discovery scheme in wireless networks, where each node is equipped with directional antennas. In [10], the authors propose an efficient broadcasting scheme using directional antenna in ad hoc wireless networks. Their scheme is based on 2-hop neighborhood information, and does not rely on location or angle information. Authors in [11] propose an efficient neighbor discovery model, which relies neither on omnidirectional antennas, nor on time synchronization. The technologies of directional antennas are the preliminaries of our algorithm, and ensure that our sense-in-order model can be improved through directional antennas.

\section{PRoblem Statement}

\section{A. Background and Motivation}

There are usually multiple channels in CRNs. Before transmission, nodes in CRNs need to find a channel to access that is available at that time. Therefore, one problem arises: which channel should be selected for sensing first? If the node happens to select a channel that is occupied by primary users for sensing, then it needs to switch to another channel and repeat the sensing again, which increases the delay and energy cost. Therefore, it is more efficient for each node to know which channels are more possible to be available. Since nodes within the close area face a similar channel situation, we consider having each node share its known channel availability information with its neighbors. These neighbors can make use of the shared information and thus have preferences on some channel when they need to pick one channel for sensing.

Besides, there are two more factors that influence the effects of the sensing results shared by other nodes:

- Valid time duration: The pattern of primary users' activities is random, which makes the spectrum availabilities dynamic. After a time period, the sensing results shared by other nodes may be invalid. Thus, there should be a valid time duration for the shared sensing results.

- Interference by secondary users: For a secondary user, although some channels are free from primary users, they may still be unavailable if they are occupied by other secondary users nearby. Thus, during the sensing phase, secondary users should also try to avoid sensing the channels that are occupied by others.

To build the order of channels for sensing on each node, the space and time domain factors need to be considered. The influences of both primary and secondary users should be considered separately.

\section{B. Problem Formulation}

We consider a CRN with $N$ nodes and $M$ channels. There is a set of primary users whose active patterns are random. When a primary user is active, nodes in its interference range are unable to use the channel that is occupied by the primary user. We assume that there is a common control channel (CCC) in the network, which is used by secondary users to share the sensing results. Each node does not sense the channel unless it needs to find a channel for transmission. Also, we assume that the sensing results of each node is accurate. we use $u$ to denote any node that shares the sensing results, and $v$ to denote any node that needs to pick up a channel for transmission.

Definition 1. A channel is sensed as available if and only if it is neither occupied by primary users nor secondary users.

When the channel is sensed as available, the secondary users can access the channel and use that for transmission. 


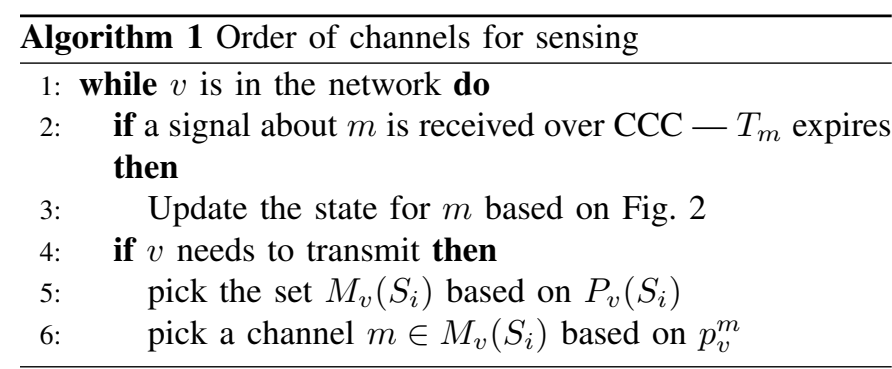

Otherwise, secondary users need to switch to another channel for sensing, which costs more energy and delay. We assume that the delay and energy cost of each switch from one channel to another channel for sensing is a constant. Therefore, we can use the following counter to identify the cost.

Definition 2. The cost $C_{v}$ of each node $v(v \in N)$ during the spectrum sensing phase is calculated as the number of switches among channels that are needed until an available one is found.

The goal of our model is to provide an order of channels for sensing so that the cost during the spectrum sensing phase is minimized. The objective can be written as: Min $\sum_{v \in N} C_{v}$.

\section{SENSE-IN-ORDER MODEL}

\section{A. Construct State Transition Diagram}

In our model, each node broadcasts its sensing results through the CCC. Based on our assumptions in Section III, each node does not sense the channel until it needs a channel for transmission. Thus, if the node finds an available channel, it will access that channel. It will also broadcast when it accesses and when it quits that channel. This can be done by broadcasting different signals. We give the following three different types of signals that can be sent by a node $u$ :

- $P O_{m}$ : channel $m$ is occupied by primary users;

- $S O_{m}$ : channel $m$ is free from primary users, but is occupied by the secondary user who sent this signal;

- $S F_{m}$ : Secondary user finishes transmission and quit from channel $m$.

To simplify our mode, we assume that there is no loss of the signal transmission over the CCC. If channel $m$ is occupied by secondary users, node $u$ will avoid sensing that channel since $S O_{m}$ was received previously by $u$ until the $S F_{m}$ is received. Based on the received signals, a node $v$ is able to identify four different states, $S=\left\{S_{i}, 1 \leq i \leq 4\right\}$, for a channel $m$. We use $<S_{i}, m>$ to indicate that channel $m$ is in state $S_{i}$ :

- $<S_{1}, m>$ : $m$ is occupied by primary users;

- $<S_{2}, m>$ : $m$ is not occupied by primary users, but is occupied by the secondary user:

- $<S_{3}, m>$ : the secondary user previously using $m$ has finished transmission and quit from $m$;

- $<S_{4}, m>$ : no signal is received about $m$.

The above four states are maintained on node $v$ itself. For $<S_{1}, m>$, node $v$ is not sure about whether the primary users have finished transmission on $m$ if no other sensing results are received from other nodes. For $\left\langle S_{2}, m\right\rangle$, node $v$ should avoid sensing $m$ until $v$ receives the signal $S F_{m}$. For $\left\langle S_{3}, m\right\rangle$, node $v$ should assign higher probabilities for selecting $m$ to sense. For $\left\langle S_{4}, m\right\rangle, v$ is not sure about the availability of $m$ either.

Furthermore, the weight of each signal about its accuracy should consider the following two issues:

- spacial domain: The channel state information that is received from the closer area is more accurate. Therefore, the channel state information that is sensed by the node itself is usually more reliable than the information shared by others.

- time domain: The message that is received more recently is usually more accurate, which is due to the dynamics of primary users' and secondary users' activities. Some channels that were available in the previous time slot may possibly become unavailable in the next time slot.

In our model, each node only collects one-hop neighbors' information and updates the channel states. Since the interference area of a primary user is usually much larger than that of a secondary user, nodes within one-hop distance may very possibly share similar information. This is normally true in real life scenarios. Therefore, there is no need to distinguish the weight of the information shared by a node's neighbors based on their distance to this node. In our simulation, we will study the influence of spacial factors.

The time domain matters more than the spacial domain. Firstly, as we explained above, the information that is received more recently is more reliable. The channel state should be updated according to the most recently received information about that channel. Secondly, the channel states may vary without being known by any node. Therefore, we add a valid time period $T$ for $S_{1}, S_{2}$, and $S_{3}$. If no signal is received about channel $m$ during $T$, node $v$ will change from $\left\langle S_{1}, m\right\rangle$, $<S_{2}, m>$, or $<S_{3}, m>$ to $<S_{4}, m>$.

The state transition diagram among different $S_{i}$ of a single channel $m$ maintained on node $v$ is shown in Fig. 2. The initial state of each channel is $S_{4}$. A state transition occurs when a signal from another node is received, or the valid time period $T$ expires. Each node maintains a state identification for each channel, and updates the state based on Fig. 2. Each state is updated based on the most recent signal in the time domain. Since primary users have higher privileges on each channel, $P O_{m}$ could be received no matter what the previous state is. State $S_{3}$ can only be reached from $S_{2}$, since we assume that there is no packet loss. Each node will mark the channel as $S_{2}$ after receiving $S O_{m}$, and will not update the state as $S_{3}$ or $S_{4}$ before $S F_{m}$ is received. Thus, there is no valid time expiration issue for state $S_{2}$.

\section{B. Sort Sensing Order}

Since each node stores the state for every channel, it now can define preferences on different channels when it needs to select one channel for sensing.

First, each node divides the whole channel set into four (at most) different subsets, based on the state of each channel. For 


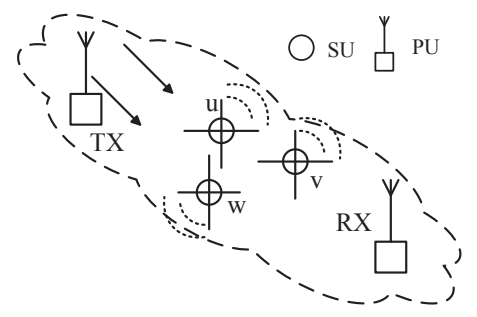

Fig. 3. Directional antenna creates more channel availabilities.

node $v$, the whole channel set $M$ is divided into four subsets $M_{v}\left(S_{i}\right), 1 \leq i \leq 4$. If channel $m \in M_{v}\left(S_{i}\right)$, channel $m$ is identified as state $S_{i}$ by node $v$. Next, we define the order or probability for each subset to be chosen. Here, the probability of a subset being chosen equals the sum of the probability that any channel in that subset will be chosen.

Definition 3. The probability of $M_{v}\left(S_{i}\right)$ being chosen is:

$$
P_{v}\left(S_{i}\right)=\frac{\left|M_{v}\left(S_{i}\right)\right| \times W_{i}}{|M|},
$$

where:

$$
\begin{gathered}
i=\{1,2,3,4\}, \\
W_{2}=0, \\
W_{3}>W_{4}>W_{1}, \\
\sum_{i}\left(\left|M_{v}\left(S_{i}\right)\right| W_{i}\right)=|M| .
\end{gathered}
$$

$\left|M_{v}\left(S_{i}\right)\right|$ denotes the number of channels in $M_{v}\left(S_{i}\right)$. (2) means that the channels identified as state $S_{2}$ will not be chosen for sensing. This is because these channels are definitely unavailable, according to the discussions in previous sections. The relationships in (3) are due to the fact that channels on state $S_{3}$ are more likely to be available than channels on $S_{1}$ and $S_{4} . W_{i}$ is the weight assigned to choosing each channel set $M_{v}\left(S_{i}\right)$. This means that, a channel is sensed to be occupied by primary users, it is still possible that it is available when sensed by node $v$, since primary users at that time may finish a transmission. (4) ensures that $\sum_{i} P_{v}\left(S_{i}\right)=1$.

For different channels in $M_{v}\left(S_{4}\right)$, the probability of each one being chosen is the same. For different channels in $M_{v}\left(S_{1}\right)$ and $M_{v}\left(S_{3}\right)$, the probability of a single channel $m$ being chosen should also be related to the amount of time that $m$ has been in that set. The more time that $m$ is in $M_{v}\left(S_{1}\right)$ or $M_{v}\left(S_{3}\right)$, the less accurate that the state of $m$ will be. Node $v$ maintains a time duration, $t_{m}$, to indicate how long $m$ has been in that sate. Then we define the probability that a single channel $m$ will be chosen:

$$
p_{v}^{m}=\left\{\begin{array}{ll}
\frac{t_{m}}{\sum_{m_{0} \in M_{v}\left(S_{1}\right)} t_{m_{0}}} \times P_{v}\left(S_{1}\right) & m \in M_{v}\left(S_{1}\right) \\
0 & m \in M_{v}\left(S_{2}\right) \\
\frac{T-t_{m}}{\sum_{m_{0} \in M_{v}\left(S_{3}\right)}\left(T-t_{m_{0}}\right)} \times P_{v}\left(S_{3}\right) & m \in M_{v}\left(S_{3}\right) \\
\frac{P_{v}\left(S_{4}\right)}{\left|M_{v}\left(S_{4}\right)\right|} & m \in M_{v}\left(S_{4}\right)
\end{array} .\right.
$$

TABLE I

SIMULATION SETTINGS

\begin{tabular}{|c|c|}
\hline number of nodes & {$[10,18]$} \\
\hline number of channels & {$[4,12]$} \\
\hline number of primary users & {$[8,14]$} \\
\hline session duration of secondary users & {$[5,10]$} \\
\hline session duration of primary users & {$[10,16]$} \\
\hline valid time period, $T$ & 20 \\
\hline$T_{S C}$ for SIO-SC & {$[0,15]$} \\
\hline$W_{3} / W_{4}$ & {$[2,3]$} \\
\hline$W_{4} / W_{1}$ & {$[1.5,2.5]$} \\
\hline
\end{tabular}

For a channel $m \in M_{v}\left(S_{1}\right)$, the longer $t_{m}$ means that $m$ is more likely to be available, since primary users have a higher probability of finishing with $m$. For a channel $m \in M_{v}\left(S_{3}\right)$, the less $t_{m}$ indicates that $m$ has a higher probability of being available. When $t_{m}$ grows larger, there is an increased possibility that $m$ may be taken by primary users without notifying node $v$.

Algorithm 1 is for a node $v$ to define the order of channels for sensing. Node $v$ keeps overhearing the CCC and monitoring if $T_{m}$ expires for each $m$. When $v$ needs to select a channel for sensing, it first decides which subset $M_{v}\left(S_{i}\right)$ to choose. Then, from the picked $M_{v}\left(S_{i}\right)$, it chooses the channel for sensing. This maintains the priorities among different $M_{v}\left(S_{i}\right)$, which are stated above. Also, since $\sum_{m \in M} p_{v}^{m}=1$, there is always one channel that can be chosen when a node senses the channel.

\section{Some Analysis}

Since nodes in a close area maintain similar states for each channel, when multiple nodes among them need to pick a channel for sensing at the same time, it is very possible for them to choose the same channel. However, in our model, the chance of conflicts can be reduced by adjusting $W_{i}$ in the expression of $P_{v}\left(S_{i}\right)$. If conflicts occur more often when choosing channels from $M_{v}\left(S_{i}\right)$, the $W_{i}$ is reduced. We will show the effects of adjusting $W_{i}$ on the whole cost in our simulation.

The probability for each channel to be chosen by a node $v$ is not only determined by $W_{i}$, but is also determined by the number of channels in the same state, $\left|M_{v}\left(S_{i}\right)\right|$. Therefore, it is not always true that the probability of the set of channels in state $S_{3}$ being chosen is higher than the set of channels in $S_{1}$ or $S_{4}$. This is compatible with the real situation. For example, when no channel is in state $S_{3}$, the probability of choosing channels from set $M_{v}\left(S_{3}\right)$ should be 0 . However, when the number of channels in each state is equal, channels in $M_{v}\left(S_{3}\right)$ are more likely to be chosen than channels in other states.

We are unable to predefine the values of $W_{1}, W_{2}$, and $W_{3}$, due to the limitations of equations (3) and (4). However, the relationship among them can be defined. Each node can then calculate the probability for each channel dynamically.

\section{IMPROVED MODEL With DiRECTIONAL ANTENNA}

We consider a model in which each secondary user is equipped with a directional antenna that is able to work in four different directions. We assume that each node can send 


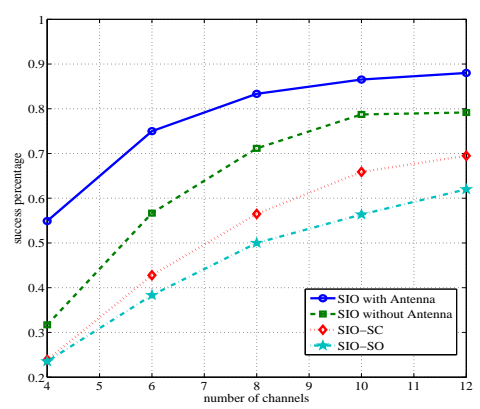

(a) change channels

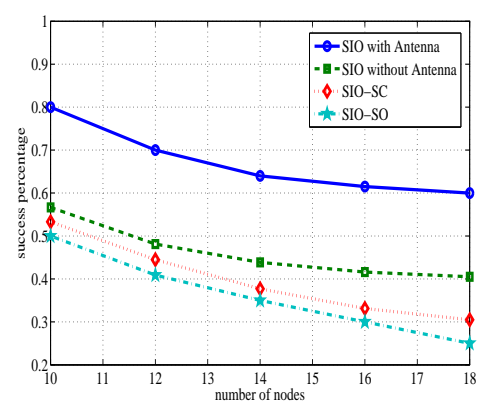

(b) change nodes

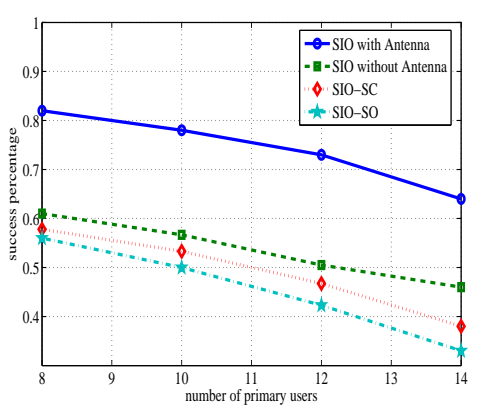

(c) change primary users

Fig. 4. Comparison of success percentage when varying network settings.

over one direction, while overhearing from four directions through the CCC on one channel. As shown in Fig. 3, there are two primary users, TX and RX, who are active and occupy a channel. The secondary users, $u, v$, and $w$, are located in the interference area of the primary users. Instead of being unable to use the channel that is occupied by the two primary users, with the directional antennas, they are now able to use that channel from one of the four directions. Therefore, the channel availabilities are improved for each secondary user.

With increased channel opportunities, the introduction of the angle dimension is problematic to our sense-in-order model. Next, we focus on how to determine whether one channel is available in one direction, based on the information provided by neighbors. For any state $S_{i}, S_{i} \in S$, we use 1 to denote that $S_{i}$ is true and 0 to denote that $S_{i}$ is false on each direction for every channel. The above state transition diagram can be easily extended here through adding two substates for each $S_{i}$ and corresponding signals on each direction. Here, we concentrate on how to determine if a single state $S_{i}$ is true or false for each direction.

For a single node, if it receives a signal of a state in one channel, the node will set the state of that channel to 1. Otherwise, it will set it to 0 . We use aaaa to denote whether a state is true in four quadrants. Each quadrant denotes a direction. Obviously, $a=0$ or 1 . The problem is for a single state, it is possible that one node can have 16 different sequences from four directions regarding the same channel. For example, 1110, 0011, etc. This is because each direction is adjacent to another while the interference area may cover part of multiple directions together. Nodes at different positions of each direction may broadcast different signals simultaneously. When one node hears different sequences within a close time period, it may be difficult to decide which direction is available.

The method we use here is a weight-based scheme, which considers both the number of signals received and the time at which the signal was received. As we explained before, the signal that is received more recently is more accurate. We use $T T$ to denote the time period since receiving the signal 1 , and $T F$ to denote the period since receiving the signal 0 , on each direction regarding each state. We also define a valid time period, $T^{\prime}$, for each signal. Signals received before $T^{\prime}$ will be ignored. Then, each node is able to calculate the probability

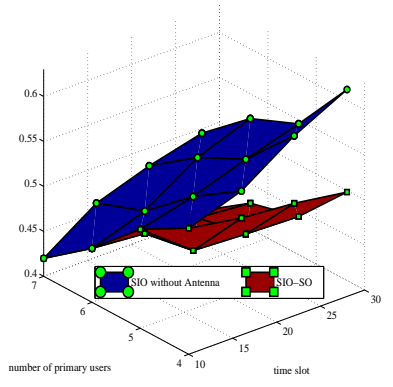

(a) Time

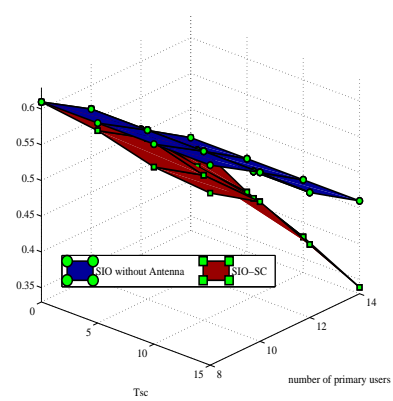

(b) $T_{S C}$
Fig. 5. Study of parameter influences

of a state to be true or false on each direction for one channel by using the following equation:

$$
\begin{gathered}
P T=\frac{\sum_{\forall T T<T^{\prime}}\left(T^{\prime}-T T\right)}{\sum_{\forall T T<T^{\prime}}\left(T^{\prime}-T T\right)+\sum_{\forall T F<T^{\prime}}\left(T^{\prime}-T F\right)} ; \\
P F=\frac{\sum_{\forall T F<T^{\prime}}\left(T^{\prime}-T F\right)}{\sum_{\forall T T<T^{\prime}}\left(T^{\prime}-T T\right)+\sum_{\forall T F<T^{\prime}}\left(T^{\prime}-T F\right)} .
\end{gathered}
$$

Since $P T+P F=1$, each node determines that a state is true on one direction for one channel if $P T>0.5$, and false if $P F \geq 0.5$. After the state is decided, the previous SIO model can be applied for each direction.

\section{Simulations}

\section{A. Simulation Settings}

We randomly generate a number of nodes which make up a one-hop network. Each node gets a randomly received session request, and stays in that session for several time slots. For a node, when a session request comes, it needs to pick a channel to sense. Also, we generate a number of primary users who randomly become active. The simulation setting parameters are shown in Table I.

Considering that the objective of our model is to minimize the number of switches among channels during spectrum sensing according to Definition 1, we measure the performance of our model using the success percentage, which is defined as the ratio between the number of times that available channels 
are sensed and the total number of times that we attempt to sense channels.

We also implement two other algorithms:

- The basic algorithm: SIO-SO(sense-in-order model with self information considered only). Nodes running SIOSO do not share their sensed channel information. Each node only uses its sensed channel information and history to decide each channel's state.

- The self weighted more algorithm: SIO-SC (sense-inorder model with self information weighted more). The SIO-SC takes the spacial factor into consideration. Each node running SIO-SC assigns more weight to the channel information sensed by itself, since it is one that is "closest" to itself. We assign a valid time window, denoted as $T_{S C}$, where $T_{S C}<T$. Any signal received from its neighbors within $T_{S C}$ regarding the same channel state information would be ignored. If any signal is received between $T_{S C}$ and $T$, the channel state would be updated according to the received signal. SIO-SC is the same as SIO without antenna when $T_{S C}=0$.

\section{B. Simulation Results}

We first compare the four algorithms, SIO with Antenna, SIO without Antenna, SIO-SC and SIO-SO. We vary the three network parameters (number of nodes, number of channels, and number of primary users) and calculate the average success percentage in the whole network. $T_{S C}$ for SIO-SC is set as 10. The results are shown in Fig. 4. We can tell that the SIO with antenna model achieves the best performance while the SIO-SO is the worst one. Also, in Fig. 4(a), the performances of all four algorithms increase when the channel number increases. In Fig. 4(b), when the number of nodes increases, the performance of all four algorithms is lowered. In Fig. 4(c), the performances of all four algorithms decrease when the amount of primary users increases.

For a better comparison, we also study the performance differences between the SIO without antenna model and the SIO-SO scheme over a time period. The SIO with antenna scheme has a similar trend as the one without antenna, which is not shown here. We vary the number of primary users and record the success percentage along a time duration. The results are in Fig. 5(a). It shows that when the primary users decrease, the performances of both schemes increase. However, the performance of the SIO-SO scheme increases more slowly than our model. The influence of $T_{S C}$ on SIO-SC is studied in Fig. 5(b). The performances of both algorithms decrease as the primary users increase. When the value of $T_{S C}$ increases, the gap between the two algorithms increases.

The influences of the algorithm parameters are shown in Fig. 6. The SIO with antenna is not shown here due to the same reason as above. We calculate the success percentage at different time slots when running the simulation. In Fig. 6(a), the value of $W_{3} / W_{4}$ is set as a constant 2.5 , while the value of $W_{4} / W_{1}$ varies in $\{1.5,2,2.5\}$. It shows that when the time increases, the performances of the three settings increase. In Fig. $6(\mathrm{~b})$, the the value of $W_{3} / W_{4}$ varies in $\{2,2.5,3\}$, while

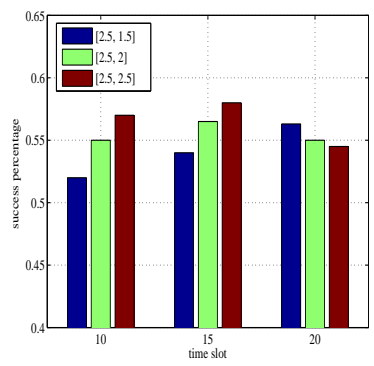

(a) change $W_{4} / W_{1}$

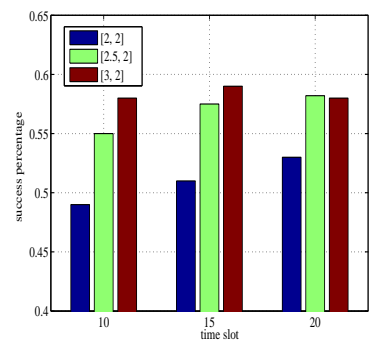

(b) change $W_{3} / W_{4}$
Fig. 6. Comparison of success percentage when varying algorithm settings.

the value of $W_{4} / W_{1}$ is set as a constant 2 . The performances of the three settings are better when the time slot increases.

\section{CONCLUSION}

In this paper, we focus on how to choose a channel for sensing for each node in cognitive radio networks (CRNs). We propose an SIO model for the pre-phase of spectrum sensing. We construct a state transition diagram and a corresponding algorithm for each node to calculate the probability of each channel being chosen for sensing. We also extend our SIO model by adding the angle dimension. We perform extensive simulations to show the performance of our model. We also implement two other algorithms for a better comparison. The simulation results demonstrate that our model outperforms the others.

\section{REFERENCES}

[1] I. F. Akyildiz, W.-Y. Lee, M. C. Vuran, and S. Mohanty, "Next generation/dynamic spectrum access/cognitive radio wireless networks: A survey," Computer Networks, 2006.

[2] T. Yucek and H. Arslan, "A survey of spectrum sensing algorithms for cognitive radio applications," IEEE Communications Surveys Tutorials, 2009.

[3] Y. Zhao, M. Song, C. Xin, and M. Wadhwa, "Spectrum sensing based on three-state model to accomplish all-level fairness for co-existing multiple cognitive radio networks," in Proc. of IEEE INFOCOM, March 2012.

[4] J. Laska, B. Bradley, T. Rondeau, K. Nolan, and B. Vigoda, "Compressive sensing for dynamic spectrum access networks: techniques and tradeoffs," in Proc. of IEEE DySPAN 11.

[5] J. Ma, G. Zhao, and Y. Li, "Soft combination and detection for cooperative spectrum sensing in cognitive radio networks," IEEE Transactions on Wireless Communications, 2008.

[6] A. Cacciapuoti, I. Akyildiz, and L. Paura, "Correlation-aware user selection for cooperative spectrum sensing in cognitive radio ad hoc networks," IEEE Journal on Selected Areas in Communications, 2012.

[7] R. Fan and H. Jiang, "Optimal multi-channel cooperative sensing in cognitive radio networks," IEEE Transactions on Wireless Communications, 2010.

[8] H. Li, "Customer reviews in spectrum: Recommendation system in cognitive radio networks," in Proc. of IEEE DySPAN 10.

[9] H. Li, "A recommendation system in cognitive radio networks with random data traffic," IEEE Transactions on Vehicular Technology, 2011.

[10] F. Dai and J. Wu, "Efficient broadcasting in ad hoc wireless networks using directional antennas," IEEE Transactions on Parallel and Distributed Systems, 2006.

[11] E. Felemban, R. Murawski, E. Ekici, S. Park, K. Lee, J. Park, and Z. Hameed, "Customer reviews in spectrum: Recommendation system in cognitive radio networks," in Proc. of IEEE Secon 10. 